\title{
Deducing the Properties Required by General Collective Intelligence Platforms
}

\author{
Andy E. Williams, Nobeah Foundation, Nairobi, Kenya
}

\begin{abstract}
A functional modeling approach is used to derive the properties that must be possessed by a platform with the capacity to significantly increase the general collective intelligence or c factor of groups. Such platforms have been termed "General Collective Intelligence" or GCI platforms. Having general problem-solving ability, a GCI potentially enables groups to execute any collective reasoning process, including abstracting (generalizing) a reasoning process so it might be reused in any other domain where it applies. A GCI can be shown to have the potential to exponentially increase the capacity of a group to create generalizations and other relationships, and capacity to store and exchange those relationships. Since relationships are concepts, and since the number of relationships between concepts better specify the location of any concept in conceptual space and therefore increases the density of conceptual space as a whole, GCI represents a phase change in collective cognition at which the collective conceptual space can expand exponentially in size and density. Each reasoning process connecting this far larger space of concepts has outcomes, making it potentially possible through these additional concepts to accumulate far greater impact on any outcome in the world. Because this phase change is not believed to have been possible at any point before in history, and is believed cannot occur again until the advent of another system with general problem-solving ability, such as a second order GCI or an Artificial General Intelligence (AGI), and because both AGI and second order GCI are believed to require GCI, GCI is proposed here to be the most important innovation in the history and immediate future of human civilization.
\end{abstract}

\section{Introduction}

Groups have been suggested to have some innate general collective intelligence that has been described as a "c factor" by analogy with the "g factor" used to describe individual intelligence [1]. Defining systems of organization that combine individuals into a "super intelligence" has also been suggested to be possible [2]. A model by which the intelligence of individuals might act in a group to create a single general collective intelligence factor has also been defined [3]. A model for a General Collective Intelligence (GCI) platform with the potential to significantly increase that general collective intelligence factor has been defined [4], but has not yet gained enough traction to be published except as a brief summary [5], though as new journals on the topic of collective intelligence continue to arise, and as the Human-Centric Functional Modeling approach this model is based on become more wellknown, publication of the model in more detail and at length in a peer reviewed journal might be on the horizon. Because it is uncertain whether a great many people and other resources will be required in order to implement a GCI, and because the way those people choose to solve the problems involved will determine what the GCI will look like, in order to be implemented this GCI has been suggested to require an iterative evolutionary approach in which some small subset of GCI functionality is built to achieve some impact, and the value of that impact used to engage a larger group to build more GCI functionality to achieve still greater impact, so that groups self-assemble in whatever self-sustaining way is required to construct a GCI. Some of the functionality required to implement a GCI, such as an extensive library of semantic models, has not yet been implemented, but approximations for all functionality have been defined in order to make it possible to achieve some of the potential increase in ability to solve collective problems that is believed to be possible through GCI [6].

\section{Properties Required by a General Collective Intelligence}

Assume that the cognition of an individual can be modeled as navigating a space of concepts or a "conceptual space" in which reasoning or understanding processes form paths between concepts. 
Assume that all concepts and all understanding as well as reasoning processes possessed by any individual can be used to construct a collective conceptual space. Defining a General Collective Intelligence or GCI to be a platform that organizes individuals in a way that enables them to converge on navigating a single coherent path though this collective conceptual space, what properties will this GCI be required to have?

Firstly, it must have general problem-solving ability and therefore the ability to perform the function of any other collective intelligence based decision-making system. Assume that solving a problem can be represented as finding a way to navigate a path from one point in conceptual space representing one concept, to another point in conceptual space representing another concept. In order for the collective cognition to reliably converge on a single coherent reasoning process, the collective cognition implemented by the GCI must be a stable function of the group. Or in other words the functionality can't spontaneously appear or disappear. We will call this requirement "functional stability". The GCI must also have the capacity to adapt in any way required to maintain this stability. We will call this requirement "functional adaptation".

Assume that fitness in solving that problem (how well that problem is solved) can be measured in some way such as by volume of outcome per unit of inputs such as cognitive effort. Assume there are a great many potential reasoning processes. In order for a GCI to be able to determine which reasoning process is the most fit, the GCI must have some mechanism for determining fitness. We will call this requirement "functional fitness". In order to be able to use this mechanism to solve any problem in general, which a system with general problem-solving ability must do, this mechanism for determining fitness must be universally applicable to any reasoning. In order to have something to measure, there must be a uniform mechanism for representing what outcome any reasoning functions to accomplish. We will call this requirement "functional modeling". With this functional modeling a GCI might define models to represent the reasoning in any other collective intelligence solution, so that all collective intelligence solutions can potentially be added to a library. With this ability to determine the fitness of each CI solution in each context, the GCI might use that library to increase its general problem-solving ability.

In order to be able to collectively use such information there must be a mechanism for defining the different contexts in which reasoning might be used, a mechanism for storing which reasoning is most fit in which contexts, and a mechanism for selecting the most fit reasoning as these domains of fitness are crossed. We will call this requirement "functional domain bridging". Assume that collectively navigating a coherent path through the collective conceptual space in order to achieve some targeted outcome requires $\mathrm{N}$ reasoning steps that might involve as many as $\mathrm{N}$ individuals to each execute one step. In order to orchestrate those $\mathrm{N}$ individuals in doing so the GCI must have a mechanism for measuring the projected fitness of each individual in executing each step of the reasoning process in order to select the individuals that are most fit. We will include this in the "functional fitness" requirement.

Collective reasoning processes might be defined by one or more people and targeted at any collective outcome. These collective reasoning processes need to have the capacity to include any reasoning or information possessed by any individual in the group. In order for this to be the case every functional model of reasoning or understanding processes must be able to be represented in terms of a common set of operations that "spans" the entire collective conceptual space. We will call this requirement "functional decomposition". In addition to defining a basic set of operations that might be used to represent any reasoning or understanding, additional operations might be defined within subdomains in order to increase ability to collectively reason within those subdomains. As an example, a functional 
model might be defined to represent the operations of a computing platform. Those operations form a subdomain of the reasoning operations that span the entire conceptual space.

In order for it to be possible for anyone in the group to find and reuse any reasoning possessed by anyone else in the group, they must be able to define a path to that reasoning process. In this representation of conceptual space a generalization of a concept is a larger region in conceptual space that includes one or more smaller (more specific) concepts. If a problem can be defined as the gap between one concept and another, a generalized problem can be defined as the gap between one generalized concept and another. By solving the problem of mapping the more specific problem to the more general problem, any solution to a specific problem satisfying that generalization can be located by its relationship to the generalization.

Creating the ability to reliably define a path to any reasoning process requires defining a concept to be sufficiently general that it relates to all other concepts, or in other words it requires the ability to collectively generalize in a way that spans the entire conceptual space. In order to do so, a GCI must have the capacity to exchange understanding not just information. That is, a GCI must have the capacity to model reasoning and information in a fully self-contained way. A fully self-contained description of meaning is a semantic model. Therefore the functional model of conceptual space, and any additional functional models defined on top of that, are expected to be complete semantic representations. A complete semantic representation of information is by itself a potentially important innovation.

In summary, the features required by a model of GCI are then as shown in table 1:

\begin{tabular}{ll}
\hline Component of Model & Description \\
Functional modelling (problems and & $\begin{array}{l}\text { Components are modelled only by function } \\
\text { to remove prejudice for or against any given } \\
\text { implementation. }\end{array}$ \\
\hline Functional decomposition & $\begin{array}{l}\text { Functional components are decomposed } \\
\text { into their most basic functional building } \\
\text { blocks for reuse. }\end{array}$ \\
\hline Functional domain bridging & $\begin{array}{l}\text { Different domains in which different } \\
\text { functions are more fit in achieving the same } \\
\text { purpose are identified. These domains are } \\
\text { bridged by using a set of weights which } \\
\text { identify the best function in each domain. }\end{array}$ \\
\hline Functional fitness & $\begin{array}{l}\text { Every functional component is assigned } \\
\text { some projected and actual fitness in } \\
\text { achieving its function. }\end{array}$ \\
\hline Functional stability & $\begin{array}{l}\text { For functional components to persist they } \\
\text { must display some degree of stability in } \\
\text { fitness to function. }\end{array}$ \\
\hline Functional adaptation & $\begin{array}{l}\text { For functional components to persist in a } \\
\text { changing environment they must have the } \\
\text { ability to adapt their function. }\end{array}$ \\
\hline
\end{tabular}

Table 1. Components of a General Collective Intelligence. 
In addition to these features, a GCI should increase intelligence over that of any individual. Representing general problem-solving ability (intelligence) as the volume of conceptual space that can be navigated per unit time, then in order to be useful a GCI should significantly increase that volume.

One way to increase the volume in conceptual space that can be navigated is to increase the number of navigable paths through conceptual space. This can be achieved by increasing capacity to generalize until generalizations can potentially span the entire conceptual space, at which point the number of reasoning paths rises exponentially with the number of concepts [7]. This in turn can be achieved by creating the capacity to store generalizations as understanding (i.e. semantically) rather than as information that must be interpreted by the listener in order to communicate an understanding.

Another way to increase the conceptual space that can be navigated is to increase the density. The resolution with which a concept might be located in the collective conceptual space is qualitatively related to the number of reasoning relationships that link it to other concepts. This in density in turn can be achieved by creating the capacity to store relationships as understanding rather than as information that must be interpreted by the listener in order to communicate an understanding.

In order to be able to scale or change outcomes of collective reasoning by executing reasoning processes in series or in parallel, and then by involving as many people as required to achieve that scale or change, collective reasoning processes must be decoupled from being aligned with any particular individual. Since software automates reasoning processes, then any software that automates this cooperation in any domain must share the common elements of functionality required to decouple processes in this way so that groups can self-assemble in any way required to maximize collective outcomes in executing those processes. This functionality has been abstracted into a proposed platform called the Peer to Peer Social Fabric (P2PSF) [8].

In order to be able to reliably muster the financial and other resources required to incentivize participation in collective reasoning, it must be reliably achievable to create more value through the collective reasoning than required to incentivize any individual's participation in that reasoning. In a GCI, patterns of solutions can potentially be generalized and reused everywhere. In the model of GCI discussed in this paper, patterns of solutions have already been defined for organizing cooperation between collective reasoning processes into networks that can significantly increase outcomes and that can potentially make those outcomes self-sustaining [9]. In order to apply such a methodology to any general problem a GCI must define processes for generalizing any process so that it can be reused in solving any problem in another domain where it applies. And as mentioned, GCI must incorporate the functionality required to enable anyone to contribute such solutions so that problem-solving doesn't become confined to the solutions chosen by any gatekeepers, and therefore potentially become bound to those centralized interests rather than being bound with the collective well-being. As mentioned, the functionality believed to be required to removed the limits to scaling cooperation has been defined [8].

In summary, a GCI must have the capacity to target any general problem, it must increase the probability of solving that problem, it must increase the magnitude of impact on that problem, and it must make problem-solving sustainable. To do so a General Collective Intelligence must remove the barriers to scaling cooperation. Any CI initiative that can't successfully form collaborations with any party having aligned interests is missing the fundamental components of GCI that are required to remove those barriers. The difference between CI and GCI is like the difference between a single AI solution and Artificial General Intelligence (AGI). A single CI solution can solve a single problem or a limited range of problems, and GCI can potentially solve any problem. Different models of GCI might be possible. But some model of GCI is required in order to meet the requirements described in this paper. And these requirements must be met in order to systematically implement the functionality 
believed to be required to exponentially increase collective capacity to generalize and to define and use conceptual relationships, in order for the group to achieve an exponential increase in general problemsolving ability (intelligence) over that of any individual. Any approach to collective decision-making that is lacking a coherent model of GCI is likely to also missing be missing these key components. And it is this exponential increase in intelligence that is what gives GCI the potential for unprecedented civilizational tranformation.

\section{Implications}

The implications of an exponential increase in general problem-solving ability are an exponential increase in ability to impact any general outcome where outcomes are limited by problem-solving ability as opposed to facing hard physical limits such as access to resources. The ability to maximize collective outcomes per program dollar in a wide range of areas of social impact requires a decision system with the capacity to do so. GCI has been suggested here to be that system. The ability to maximize progress in research in a discipline such as in physics requires the ability to collectively make the most of all ideas. Considering all implications of all theories on all other theories however implies a significant increase in capacity to execute collective reasoning for such a volume of reasoning to be possible. Considering all such implications also in turn requires a common model for information and reasoning.

In Computer Engineering and Science several working papers explore how GCI might be applied to the various computing methods in order to increase collective outcomes in the use of computing [10]. In physics, the "conceptual space" used by GCI to store semantic models of concepts and the reasoning required to navigate between them is a "functional state space". All functional state spaces are semantic models in that they are fully self-contained descriptions of meaning, and are believed to share common properties in order to be self-describing, so that implementing one might be used to implement all others. Implementing a functional state space to represent the physical universe and having a semantic model of the universe potentially enables all data from any physics experiment to potentially be stored in the same data model. This creates the possibility of testing any theory on all data simply by defining a semantic model of the theory [11]. In mathematics, defining a functional state space for mathematics creates the potential to radically increase the rate at which theorems or other relationships can be discovered. Implementing this functional state space might dedicate a number of great mathematical minds towards implementing a functional state space that can be reused to implement the conceptual space. In psychology GCI involves a model of cognition and a model of collective cognition that can potentially be used in solving problems of cognition. In economics, the implications of GCI must be researched in preparation for all phases of any GCI based program, such as the proposed Collective Intelligence based Program to Accelerate Achievement of the Sustainable Development Goals (CIPAASDGs) [12]. One economic implication is the application of General Collective Intelligence or GCI to the future of work. When applied to the future of work, GCI has the unique potential to reverse the impact of automation from being a cause of job loss and increasing inequality, to being a source of job creation and increased collective financial well-being. In law, the implications of GCI must be researched in preparation for all phases of the CIPAA-SDGs program as well. Since GCI also has the potential to radically accelerate the exchange of intellectual and other property, and to create new forms of fractional ownership. This necessitates research on the legal, technical, and other barriers involved in ownership that can be divided into small fractions and exchanged in an automated way at extremely high speed and scale. Therefore the application of GCI to the law must be considered.

GCI has the potential to fundamentally change the way science is done by enabling groups to selfassemble into networks able to execute processes that maximize collective outcomes. In computational social science for example, GCI has the potential to not just predict outcomes, but also to identify 
causes by modeling causes and then detecting those models. GCI also defines a semantic model for data and reasoning, so that data and theories of causation gathered by any single sociology researcher might be leveraged by any other sociology researcher in an automated way. So by creating this "social simulation" infrastructure, it might become possible to eventually test sociological theories on all existing data by simply modeling the theories. In this GCI model each individual might interact with the GCI directly, or might interact with the GCI using an intelligent agent acting on their behalf. This intelligent agent is a model of the individual, which introduces the possibility of simulating the outcome of entire social policies based on models of every single individual in a population, and to do so in real-time in order to optimize policies. Since this simulation and testing infrastructure is potentially common to all GCI based testing and simulation, it might be reusable in research in physics, psychology, or any other subject as well.

In short, this analysis suggests the exceedingly bold claim that GCI is the most important innovation in the history of human civilization. But perhaps due to the unorthodox approach a thorough examination of the approach remains to be published. A quick survey of the literature suggests that no one other than its author is likely to be aware of the work. This reveals a certain lack of collective intelligence in the scientific process. If GCI truly is that important, then for every problem, GCI might have the single biggest potential for impact on that problem, and therefore be the most important project to collectively implement. But certain systematic factors might prevent this from happening. For example, being commercial entities businesses might be more aligned with competing as opposed to cooperation to increase mutual outcomes, and as a result might have little incentive to undertake such research. Such research might then have to be undertaken by academics since they have access to public funding. Solving the problem of letting enough academics know about GCI that it is possible to get them to apply for the research funding to actually build a GCI requires building mind share. As specific examples provided in various preprints of papers suggest, GCI has the potential to increase social impact per program dollar by orders of magnitude, but anecdotal evidence suggests GCI might be too complex and abstract to ever be funded by any social impact donors. Furthermore, the developer of this model of GCI is not an academic with a traditional academic background. Not having a PhD, it is difficult for such individuals to become affiliated with a university. Not being affiliated with a university it is difficult for such individuals to access funding to do the research themselves, therefore they must inspire others to do so. This is no small feat, since not being affiliated with a university might often be associated with "fringe science", an association that might make it very difficult to even disseminate such a disruptive idea that makes such a bold claim. It would seem impossible that an idea could be true if it is so soundly ignored, but it is precisely the lack of ability to detect situations in which some ideas are systematically ignored for reasons other than merit, that has always led to valuable ideas in science being ignored for decades or even centuries.

\section{Conclusions}

A collective cognition is represented as being created when group thinking is constrained by a function that optimizes some collective outcome. This collective cognition navigates a collective conceptual space. By analysis of this space, an argument has been made in this paper that through meeting specific conditions a GCI might exponentially increase the navigable volume of conceptual space, and therefore exponentially increase collective intelligence. An analysis in conceptual space also suggests that no other innovation in the history or future of human civilization, not even quantum computing, with the exception of Artificial General Intelligence, is likely to have equal or greater impact, and therefore equal or greater importance

\section{References}


[1] Journal article: Woolley, Anita Williams; Chabris, Christopher F.; Pentland, Alex; Hashmi, Nada; Malone, Thomas W. (29 October 2010). "Evidence for a Collective Intelligence Factor in the Performance of Human Groups". Science. 330 (6004): 686-688. Bibcode:2010Sci...330..686W. doi:10.1126/science.1193147. PMID 20929725. S2CID 74579.

[2] Book: Malone, T. W. (2018). Superminds: The surprising power of people and computers thinking together.

[3] Journal article: Krafft, P.M. (2019), A Simple Computational Theory of General Collective Intelligence. Top Cogn Sci, 11: 374-392. https://doi.org/10.1111/tops.12341.

[4] Pre-print: Williams, A. E. (2020, April 30). A Model for General Collective Intelligence. https://doi.org/10.31730/osf.io/6u984

[5] Williams A.E. (2019) The Relationship Between Collective Intelligence and One Model of General Collective Intelligence. In: Nguyen N., Chbeir R., Exposito E., Aniorté P., Trawiński B. (eds) Computational Collective Intelligence. ICCCI 2019. Lecture Notes in Computer Science, vol 11683. Springer, Cham. https://doi.org/10.1007/978-3-030-28377-3 49

[6] Williams, A. E. (2021, January 11). Approximating an Artificial General Intelligence or a General Collective Intelligence. https://doi.org/10.31730/osf.io/zsbfe

[7] Andy E. Williams, Monika Dos Santos, Emir Haliki, (2020, July 11). Human Intelligence and General Collective Intelligence as Phase Changes in Animal Intelligence.

https://doi.org/10.31234/osf.io/dr8qn

[8] Williams, A. E. (2020, December 16). The Peer to Peer Social Fabric as a Platform for General Collective Intelligence. https://doi.org/10.31730/osf.io/qbxfr

[9] Williams, A. E. (2020, December 16). Discovering and Implementing Self-Sustaining Networks of Cooperation with General Collective Intelligence. https://doi.org/10.31730/osf.io/safxk

[10] Andy E. Williams, Is General Collective Intelligence the Most Important Direction of Research in Computing Today, working paper (2021)

[11] Williams, A. E. (2020, December 18). Increasing Discovery in Research, Design, and Other Processes with Artificial General Intelligence and General Collective Intelligence.

https://doi.org/10.31730/osf.io/gz385

[12] Williams, A. E. (2020, April 30). The Collective Intelligence based Program to Accelerate Achievement of the Sustainable Development Goals as a Case Study for Collectively Intelligent Program Design. https://oi.org/10.31235/osf.io/r2dxq 